IRA-International Journal of Applied Sciences ISSN 2455-4499; Vol.04, Issue 01 (2016)

Institute of Research Advances

http://research-advances.org/index.php/IRAJAS

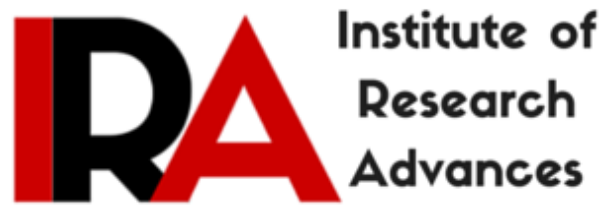

\title{
Microwave Induced Synthesis of Pyrazoline Compounds Containing Substituted Benzyloxy Phenyl Ring System
}

\author{
${ }^{1}$ Jyoti Prajapati, ${ }^{2}$ Mangalshree Dulawat, ${ }^{3}$ Prakash Prajapat, ${ }^{4}$ Renu Rathore \\ \& ${ }^{5}$ Shiv S. Dulawat
}

Department of Chemistry,

Pacific Academy of Higher Education and Research University,

Udaipur-313001, Rajasthan, India.

DOI: $\underline{\text { http://dx.doi.org/10.21013/jas.v4.n1.p17 }}$

How to cite this paper:

Prajapati, J., Dulawat, M., Prajapat, P., Rathore, R., \& Dulawat, S. (2016).

Microwave Induced Synthesis of Pyrazoline Compounds Containing Substituted

Benzyloxy Phenyl Ring System. IRA-International Journal of Applied Sciences (ISSN

2455-4499), 4(1). doi:http://dx.doi.org/10.21013/jas.v4.n1.p17

(C) Institute of Research Advances

(cc) EY-NC

This works is licensed under a Creative Commons Attribution-Non Commercial 4.0 International License subject to proper citation to the publication source of the work.

Disclaimer: The scholarly papers as reviewed and published by the Institute of Research Advances (IRA) are the views and opinions of their respective authors and are not the views or opinions of the IRA. The IRA disclaims of any harm or loss caused due to the published content to any party. 
IRA-International Journal of Applied Sciences

\section{ABSTRACT}

Green chemistry uses highly efficient and environmental benign synthetic procedures to synthesize various bioactive heterocyclic frameworks which are the useful synthons for the synthesis of medicines, plastics, petrochemicals, agrochemicals, cosmetics and many more hence the green chemistry is the need of the day. In this methodology pyrazolines have been synthesized under microwave irradiation ${ }^{l}$ using ethanol /alumina. The structures of these compounds were established by elemental analysis and spectral data. The method has several advantages in comparison with conventional synthesis including clean reaction procedure, easy workup, and short reaction time giving excellent yields of product.

Keywords: Chalcones, Pyrazolines, Basic alumina, Benzyloxy phenyl ring, Microwave irradiation.

INTRODUCTION-The use of microwaves in organic synthesis (Microwave Induced Organic Reaction Enhancement (MORE) $)^{2-3}$ has increased dramatically in the last years, receiving widespread acceptance and becoming an indispensable tool. In organic synthesis microwave technology has become a powerful tool, since by employing this technique it is generally possible to prepare organic compounds very fast, with better yields and high purity compared to other more conventional methods. Synthesis of pyrazoline is of current interest because of their broad spectrum of biological activity as antibacterial, antimycobacterial ${ }^{4}$, antiinflammator, ${ }^{5}$ antidepressant, ${ }^{6}$ anticancer, ${ }^{7}$ pesticidal, ${ }^{8}$ analgesic, ${ }^{9}$ insecticidal, ${ }^{10}$ antidiabitic, antipyretic, ${ }^{11}$ herbicidal, ${ }^{12}$ fungicidal, ${ }^{13}$ and antiviral properties. Certain pyrazoline derivatives has also shown fluorescence properties and is extensively used as optical preservatives (specially in preserving fish sausages). Therefore we focused on synthesis of some pyrazoline system. In this paper we report the synthesis of some pyrazoline derivatives containing substituted benzyloxy phenyl ring system.

\section{MATERIALS AND METHODS}

Experimental Section:- Melting points were determined in an open capillary tube and are uncorrected. IR spectra $\left(v_{\max }\right.$ in $\mathrm{cm}^{-1}, \mathrm{KBr}$ ) were recorded on a Perkin-Elmer 16pc (FTIR) spectrophotometer. Mass spectra were taken on a Jeol D-300 (EI) and VG-70S mass spectrometer and ${ }^{1} \mathrm{H}$ NMR was recorded on $\mathrm{CDCl}_{3}$ on a Varian CFT-20 or Brucker DRX-300 (300 MHz) spectrometer using TMS as internal standard (chemical shifts in $\square$, ppm) All compounds gave satisfactory elemental analysis and spectral data. All the reactions were carried out in a domestic microwave oven. (Kenstar, output energy 1200W, frequency $2450 \mathrm{MHz}$, model no. MO9706).

General procedure for the synthesis of 3-(p-chlorophenyl)-5-[4'-(p-chlorobenzyloxy)-3'methoxyphenyl]-1-phenyl-4,5-dihydro-1H-pyrazole(C-I)

The synthesis of 1-(p-chlorophenyl)-3-[4'-(p-chlorobenzyloxy)-3'-methoxyphenyl]-propenone (B) was performed using 4-(p-Chlorobenzyloxy)-3-methoxybenzaldehyde (A) and 4'-chloroacetophenone using ethanol/alumina under microwave irradiation as reported . Further the pyrazoline derivatives were carried out by the three following methods:-

(a)Conventional (Classical) Method-To a mixture of synthesized chalcone (0.005mole), phenyl hydrazine $(0.01 \mathrm{~mole}), \mathrm{EtOH}(40 \mathrm{ml}), 4 \mathrm{~N}$ solution of sodium hydroxide $(3 \mathrm{ml})$ was added and refluxed for a period of 7-8 hrs. The progress of reaction was monitored by TLC using benzene: ethyl acetate mixture $[9: 1 \mathrm{v} / \mathrm{v}]$ as eluent. The reaction mixture was cooled diluted with water and kept under refrigeration. The separated compound was filtered and recrystallised from suitable solvent.

(b)Microwave Induced Solution Phase Method-A mixture of synthesized chalcone (0.005mole), phenyl hydrazine $(0.01 \mathrm{~mole})$, ethanol $(25 \mathrm{ml})$ and $4 \mathrm{~N}$ solution of sodium hydroxide $(3 \mathrm{ml})$ was taken in a $100 \mathrm{ml}$ borosil flask fitted with a funnel as a loose top. The reaction mixture was subjected to microwave irradiation for 6-7 min, at 30\% microwave power (300W) with short interruption of $40 \mathrm{sec}^{-1} \mathrm{~min}$. to avoid the excessive evaporation of the solvent. This protocol was repeated until an overall heating time. The 
progress of reaction was monitored by TLC. The reaction mixture was cooled, diluted with cold water and kept under refrigeration. The separated product was filtered, washed with water, dried and recrystallised with proper solvent.

(c)Microwave Induced Solid Phase Method $\left(\mathbf{A l}_{2} \mathbf{O}_{3}\right)$ - A mixture of synthesized chalcone $(0.01 \mathrm{~mol}$.), phenyl hydrazine $(0.02 \mathrm{~mol})$ was dissolved in ethanol $(10 \mathrm{ml})$ and taken in a $100 \mathrm{ml}$ borosil flask. To this $4 \mathrm{gm}$ of basic alumina $\left(\mathrm{Al}_{2} \mathrm{O}_{3}\right)$ was added and the reactant and were properly mixed with the help of a glass rod. Adsorbed material was dried in air and irradiated inside the microwave oven at 5-6 minute, medium power level $(600 \mathrm{~W})$. On completion of the reaction (TLC examination), the mixture was cooled at room temperature and then product was extracted into ethanol.

REACTION SCHEME

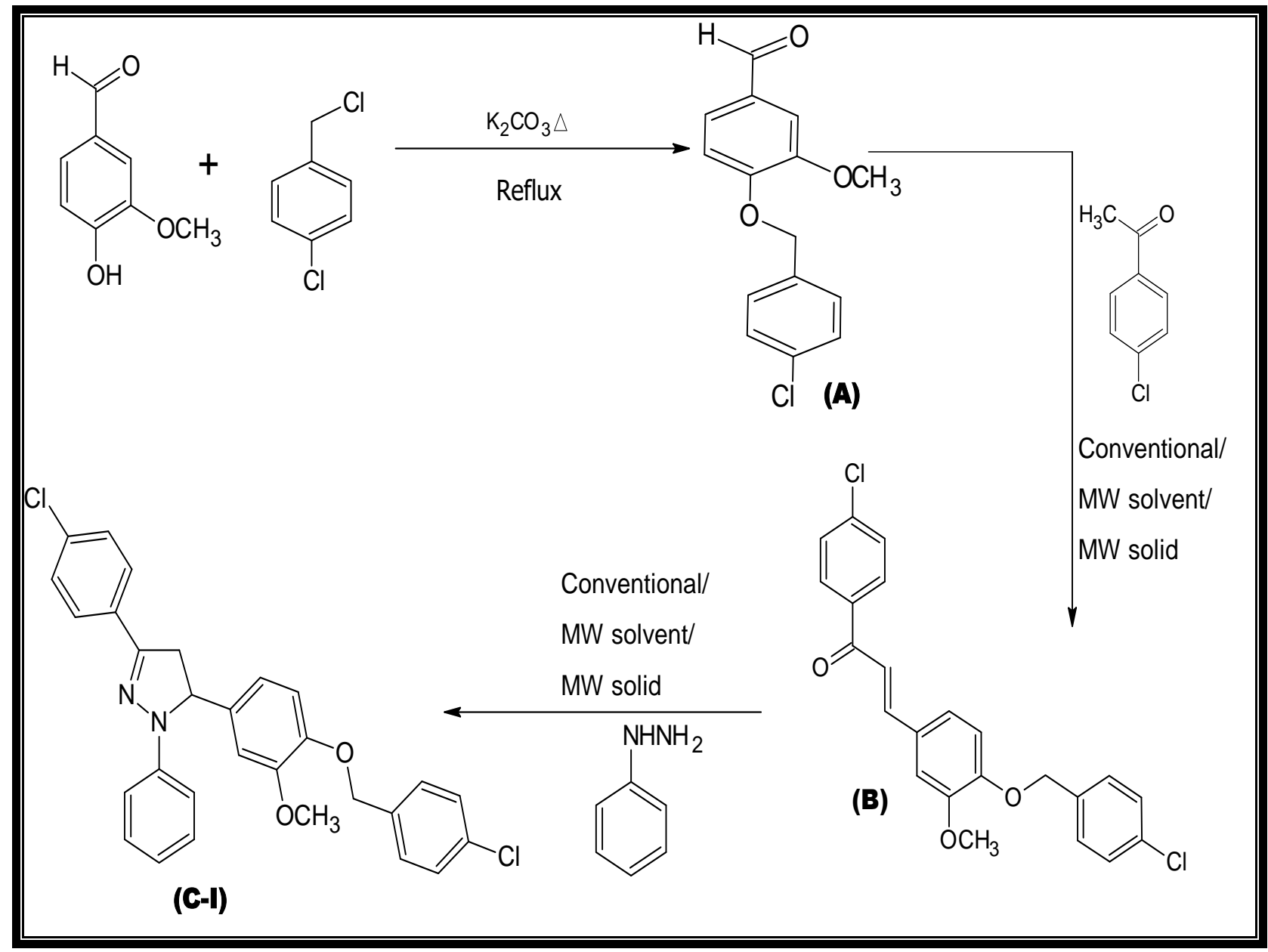


Molecular formula of synthesized pyrazoline derivatives (C-I) to ( C-V).

\begin{tabular}{|l|l|l|l|l|}
\hline Comp. No. & $\begin{array}{l}\text { Actophenone } \\
\text { Derivatives }\end{array}$ & $\begin{array}{l}\text { Substitute } \\
\text { Group } \\
\text { Actophenone } \\
\text { Derivatives }\end{array}$ & $\begin{array}{l}\text { Synthesized } \\
\text { Chalcones }\end{array}$ & $\begin{array}{l}\text { Synthesized } \\
\text { Pyrazolines Derivatives } \\
\text { (C-I) to( C-V) }\end{array}$ \\
\hline$($ C-I) & $\mathrm{C}_{8} \mathrm{H}_{7} \mathrm{ClO}$ & $\mathrm{Cl}$ & $\mathrm{C}_{23} \mathrm{H}_{18} \mathrm{Cl}_{2} \mathrm{O}_{3}$ & $\mathrm{C}_{29} \mathrm{H}_{24} \mathrm{Cl}_{2} \mathrm{~N}_{2} \mathrm{O}_{2}$ \\
\hline$($ C-II $)$ & $\mathrm{C}_{8} \mathrm{H}_{7} \mathrm{BrO}$ & $\mathrm{Br}$ & $\mathrm{C}_{23} \mathrm{H}_{18} \mathrm{BrClO}_{3}$ & $\mathrm{C}_{9} \mathrm{H}_{24} \mathrm{BrClN}_{2} \mathrm{O}_{2}$ \\
\hline$($ C-III) & $\mathrm{C}_{8} \mathrm{H}_{8} \mathrm{O}$ & $\mathrm{H}$ & $\mathrm{C}_{23} \mathrm{H}_{19} \mathrm{ClO}_{3}$ & $\mathrm{C}_{29} \mathrm{H}_{25} \mathrm{ClN}_{2} \mathrm{O}_{2}$ \\
\hline$($ C-IV) & $\mathrm{C}_{9} \mathrm{H}_{10} \mathrm{O}$ & $\mathrm{CH}$ & $\mathrm{C}_{24} \mathrm{H}_{21} \mathrm{ClO}_{3}$ & $\mathrm{C}_{30} \mathrm{H}_{27} \mathrm{ClN}_{2} \mathrm{O}_{2}$ \\
\hline (C-V) & $\mathrm{C}_{9} \mathrm{H}_{10} \mathrm{O}_{2}$ & $\mathrm{OCH}_{3}$ & $\mathrm{C}_{24} \mathrm{H}_{21} \mathrm{ClO}_{4}$ & $\mathrm{C}_{30} \mathrm{H}_{27} \mathrm{ClN}_{2} \mathrm{O}_{3}$ \\
\hline
\end{tabular}

Physical characterization of synthesized pyrazoline derivatives (C-I) to( C-V).

\begin{tabular}{|c|c|c|c|c|c|c|c|c|}
\hline \multirow[t]{2}{*}{$\begin{array}{l}\text { Comp. } \\
\text { No. }\end{array}$} & \multirow[t]{2}{*}{ Molecular formula } & \multirow[t]{2}{*}{ M.W. } & \multirow[t]{2}{*}{$\begin{array}{l}\text { M.P. } \\
\left({ }^{\circ} \mathrm{C}\right)\end{array}$} & \multicolumn{5}{|c|}{$\begin{array}{l}\text { Elemental analysis } \\
\text { Calculated/ Found }\end{array}$} \\
\hline & & & & $\% \mathrm{C}$ & $\% \mathbf{H}$ & $\% \mathrm{Cl}$ & $\% 0$ & $\mathbf{N} \%$ \\
\hline (C-I) & $\mathrm{C}_{29} \mathrm{H}_{24} \mathrm{Cl}_{2} \mathrm{~N}_{2} \mathrm{O}_{2}$ & $\begin{array}{l}503.42 \\
(503.21)\end{array}$ & 153 & $\begin{array}{l}69.19 \\
68.68\end{array}$ & $\begin{array}{l}4.81 \\
4.45\end{array}$ & $\begin{array}{l}14.08 \\
14.3\end{array}$ & $\begin{array}{l}6.36 \\
6.20\end{array}$ & $\begin{array}{l}5.56 \\
5.23\end{array}$ \\
\hline (C-II) & $\mathrm{C}_{2}{ }_{9} \mathrm{H}_{24} \mathrm{BrClN}_{2} \mathrm{O}_{2}$ & $\begin{array}{l}547.87 \\
(546.92)\end{array}$ & 197 & $\begin{array}{l}63.58 \\
63.08\end{array}$ & $\begin{array}{l}4.42 \\
4.50\end{array}$ & $\begin{array}{l}6.47 \\
6.23\end{array}$ & $\begin{array}{l}5.84 \\
5.76\end{array}$ & $\begin{array}{l}5.11 \\
4.98\end{array}$ \\
\hline (C-III) & $\mathrm{C}_{29} \mathrm{H}_{25} \mathrm{ClN}_{2} \mathrm{O}_{2}$ & $\begin{array}{l}468.97 \\
(467.34)\end{array}$ & 173 & $\begin{array}{l}74.27 \\
73.99\end{array}$ & $\begin{array}{l}5.37 \\
5.10\end{array}$ & $\begin{array}{l}7.56 \\
7.34\end{array}$ & $\begin{array}{l}6.82 \\
6.70\end{array}$ & $\begin{array}{l}5.97 \\
5.68\end{array}$ \\
\hline (C-IV) & $\mathrm{C}_{30} \mathrm{H}_{27} \mathrm{ClN}_{2} \mathrm{O}_{2}$ & $\begin{array}{l}483.00 \\
(482.56)\end{array}$ & 148 & $\begin{array}{l}74.60 \\
74.45\end{array}$ & $\begin{array}{l}5.63 \\
5.55\end{array}$ & $\begin{array}{l}7.34 \\
7.12\end{array}$ & $\begin{array}{l}6.63 \\
6.30\end{array}$ & $\begin{array}{l}5.80 \\
5.67\end{array}$ \\
\hline$(\mathrm{C}-\mathrm{V})$ & $\mathrm{C}_{30} \mathrm{H}_{27} \mathrm{ClN}_{2} \mathrm{O}_{3}$ & $\begin{array}{l}499.00 \\
(498.67)\end{array}$ & 162 & $\begin{array}{l}72.21 \\
71.87\end{array}$ & $\begin{array}{l}5.45 \\
5.07\end{array}$ & $\begin{array}{l}7.10 \\
6.78\end{array}$ & $\begin{array}{l}9.62 \\
9.51\end{array}$ & $\begin{array}{l}5.61 \\
5.56\end{array}$ \\
\hline
\end{tabular}


IRA-International Journal of Applied Sciences

Experimental data of synthesized pyrazoline derivatives (C-I) to( C-V).

\begin{tabular}{|c|c|c|c|c|c|c|}
\hline \multirow{3}{*}{$\begin{array}{l}\text { Comp. } \\
\text { No. }\end{array}$} & \multicolumn{3}{|l|}{ Reaction Time } & \multicolumn{3}{|l|}{ \% Yield } \\
\hline & \multirow{2}{*}{$\begin{array}{l}\text { Classical Method } \\
\text { (Hrs) } \\
\text { (a) }\end{array}$} & \multicolumn{2}{|c|}{ MW Methods (Min) } & \multirow{2}{*}{$\begin{array}{l}\text { Classical } \\
\text { Method } \\
\text { (a) }\end{array}$} & \multicolumn{2}{|c|}{ MW Methods } \\
\hline & & (b) & (c) & & (b) & (c) \\
\hline (C-I) & $7-8$ & $6-7$ & $5-6$ & 69 & 85 & 75 \\
\hline (C-II) & 7.5 & $6-8$ & $6-7$ & 67 & 91 & 80 \\
\hline (C-III) & $6-7$ & $5-7$ & $6-7$ & 70 & 90 & 76 \\
\hline (C-IV) & 8 & $5-6$ & $5-6$ & 65 & 88 & 74 \\
\hline$(\mathrm{C}-\mathrm{V})$ & 7 & $5-7$ & $5-6$ & 70 & 90 & 80 \\
\hline
\end{tabular}

\section{SPECTRAL ANALYSIS OF SYNTHESIZED COMPOUNDS}

(C-I) 3-(p-chlorophenyl)-5-[4'-(p-chlorobenzyloxy)-3'-methoxyphenyl]-1-phenyl-4,5-dihydro-1Hpyrazole :-IR $\vee \max (\mathrm{KBr}) \mathrm{cm}^{-1}: 3338(\mathrm{~N}-\mathrm{H}), 1682(\mathrm{C}=\mathrm{N}), 1596(\mathrm{C}=\mathrm{C}), 1233(\mathrm{C}-\mathrm{N}), 1053\left(-\mathrm{OCH}_{3}\right) .{ }^{1} \mathrm{H}$ NMR $\left(\mathrm{CDCl}_{3}, \delta \mathrm{ppm}\right): 5.07$ [s, 2H, $\left.\mathrm{OCH}_{2}\right], 3.88\left[\mathrm{~s}, 3 \mathrm{H}, \mathrm{OCH}_{3}\right], 3.06$ [dd, J=16.99 Hz,1H, Ha], 3.72 [dd, $\mathrm{J}=16.96 \mathrm{~Hz}, 1 \mathrm{H}, \mathrm{Hb}], 5.01[\mathrm{dd}, 1 \mathrm{H}, \mathrm{Hx}] 6.97-7.85[\mathrm{~m}, 16 \mathrm{H}, \mathrm{Ar}-\mathrm{H}] . \mathrm{MS}(\mathrm{m} / \mathrm{z}): 503[\mathrm{M}]^{+}, 468$ $\left[\mathrm{C}_{29} \mathrm{H}_{25} \mathrm{ClN}_{2} \mathrm{O}_{2}\right]^{+}, 427\left[\mathrm{C}_{23} \mathrm{H}_{20} \mathrm{Cl}_{2} \mathrm{~N}_{2} \mathrm{O}_{2}\right]^{+}, 378\left[\mathrm{C}_{22} \mathrm{H}_{19} \mathrm{~N}_{2} \mathrm{O}_{2}\right]^{+}, 270\left[\mathrm{C}_{18} \mathrm{H}_{22} \mathrm{O}\right]^{+}, 262\left[\mathrm{C}_{15} \mathrm{H}_{15} \mathrm{ClO}_{2}\right]^{+}, 258$ $\left[\mathrm{C}_{16} \mathrm{H}_{15} \mathrm{O}\right]^{+}, 157\left[\mathrm{C}_{10} \mathrm{H}_{21} \mathrm{O}\right]^{+}, 142\left[\mathrm{C}_{7} \mathrm{H}_{7} \mathrm{ClO}\right]^{+}, 140\left[\mathrm{C}_{8} \mathrm{H}_{9} \mathrm{Cl}\right]^{+}, 138\left[\mathrm{C}_{8} \mathrm{H}_{10} \mathrm{O}_{2}\right]^{+}, 112\left[\mathrm{C}_{6} \mathrm{H}_{5} \mathrm{Cl}\right]^{+}, 70$ $\left[\mathrm{C}_{3} \mathrm{H}_{6} \mathrm{~N}_{2}\right]^{+}$.

(C-II) 3-(p-bromophenyl)-5-[4'-(p-chlorobenzyloxy)-3'-methoxyphenyl]-1-phenyl-4,5-dihydro-1Hpyrazole :-IR $\vee \max (\mathrm{KBr}) \mathrm{cm}^{-1}: 3354(\mathrm{~N}-\mathrm{H}), 1670(\mathrm{C}=\mathrm{N}), 1585(\mathrm{C}=\mathrm{C}), 1236(\mathrm{C}-\mathrm{N}), 1072\left(-\mathrm{OCH}_{3}\right) .{ }^{1} \mathrm{H}$ NMR ( $\left.\mathrm{CDCl}_{3}, \delta \mathrm{ppm}\right): 5.05$ [s, 2H, $\mathrm{OCH}_{2}$ ] 3.82 [s, 3H, $\mathrm{OCH}_{3}$ ], 3.03 [dd, J=17.00 Hz,1H, Ha], 3.76 [dd, $\mathrm{J}=16.90 \mathrm{~Hz}, 1 \mathrm{H}, \mathrm{Hb}], 5.10[\mathrm{dd}, 1 \mathrm{H}, \mathrm{Hx}], 6.87-7.75[\mathrm{~m}, 16 \mathrm{H}, \operatorname{Ar}-\mathrm{H}] . \mathrm{MS}(\mathrm{m} / \mathrm{z}): 548[\mathrm{M}]^{+}, 471$ $\left[\mathrm{C}_{24} \mathrm{H}_{20} \mathrm{BrClN}_{2} \mathrm{O}_{2}\right]^{+}, 468\left[\mathrm{C}_{29} \mathrm{H}_{25} \mathrm{ClN}_{2} \mathrm{O}_{2}\right]^{+}, 423\left[\mathrm{C}_{22} \mathrm{H}_{19} \mathrm{BrN}_{2} \mathrm{O}_{2}\right]^{+}, 270\left[\mathrm{C}_{18} \mathrm{H}_{22} \mathrm{O}\right]^{+}, 262\left[\mathrm{C}_{15} \mathrm{H}_{15} \mathrm{ClO}_{2}\right]^{+}$, $303\left[\mathrm{C}_{16} \mathrm{H}_{15} \mathrm{BrO}\right]^{+}, 185\left[\mathrm{C}_{8} \mathrm{H}_{9} \mathrm{Br}\right]^{+}, 157\left[\mathrm{C}_{10} \mathrm{H}_{21} \mathrm{O}\right]^{+}, 142\left[\mathrm{C}_{7} \mathrm{H}_{7} \mathrm{ClO}^{+}, 138\left[\mathrm{C}_{8} \mathrm{H}_{10} \mathrm{O}_{2}\right]^{+}, 112\left[\mathrm{C}_{6} \mathrm{H}_{5} \mathrm{Cl}\right]^{+}, 70\right.$ $\left[\mathrm{C}_{3} \mathrm{H}_{6} \mathrm{~N}_{2}\right]^{+}$.

(C-III) 3-phenyl-5-[4'-(p-chlorobenzyloxy)-3'-methoxyphenyl]-1-phenyl-4,5-dihydro-1H-pyrazole :-IR $v \max (\mathrm{KBr}) \mathrm{cm}^{-1}: 3342(\mathrm{~N}-\mathrm{H}), 1667(\mathrm{C}=\mathrm{N}), 1588(\mathrm{C}=\mathrm{C}), 1238(\mathrm{C}-\mathrm{N}), 1064\left(-\mathrm{OCH}_{3}\right) .{ }^{1} \mathrm{H}$ NMR

$\left(\mathrm{CDCl}_{3}, \delta \mathrm{ppm}\right): 5.11\left[\mathrm{~s}, 2 \mathrm{H}, \mathrm{OCH}_{2}\right], 3.80\left[\mathrm{~s}, 3 \mathrm{H}, \mathrm{OCH}_{3}\right.$ ], 3.04 [dd, J=16.98 Hz ,1H, Ha], 3.74 [dd, $\mathrm{J}=16.94 \mathrm{~Hz}, 1 \mathrm{H}, \mathrm{Hb}], 5.10[\mathrm{dd}, 1 \mathrm{H}, \mathrm{Hx}], 6.88-7.93[\mathrm{~m}, 17 \mathrm{H}, \mathrm{Ar}-\mathrm{H}] . \mathrm{MS}(\mathrm{m} / \mathrm{z}): 469[\mathrm{M}]^{+}, 468$ $\left[\mathrm{C}_{29} \mathrm{H}_{25} \mathrm{ClN}_{2} \mathrm{O}_{2}\right]^{+}, 392\left[\mathrm{C}_{23} \mathrm{H}_{21} \mathrm{ClN}_{2} \mathrm{O}_{2}\right]^{+}, 344\left[\mathrm{C}_{22} \mathrm{H}_{20} \mathrm{~N}_{2} \mathrm{O}_{2}\right]^{+}, 270\left[\mathrm{C}_{18} \mathrm{H}_{22} \mathrm{O}\right]^{+}, 262\left[\mathrm{C}_{15} \mathrm{H}_{15} \mathrm{ClO}_{2}\right]^{+}, 224$ $\left[\mathrm{C}_{16} \mathrm{H}_{18} \mathrm{O}\right]^{+}, 157\left[\mathrm{C}_{10} \mathrm{H}_{21} \mathrm{O}\right]^{+}, 142\left[\mathrm{C}_{7} \mathrm{H}_{7} \mathrm{ClO}\right]^{+}, 138\left[\mathrm{C}_{8} \mathrm{H}_{10} \mathrm{O}_{2}\right]^{+}, 112\left[\mathrm{C}_{6} \mathrm{H}_{5} \mathrm{Cl}\right]^{+}, 106\left[\mathrm{C}_{8} \mathrm{H}_{10}\right]^{+}, \quad 70$ $\left[\mathrm{C}_{3} \mathrm{H}_{6} \mathrm{~N}_{2}\right]^{+}$.

(C-IV) 3-(p-methylphenyl)-5-[4'-(p-chlorobenzyloxy)-3'-methoxyphenyl]-1-phenyl-4,5-dihydro-1Hpyrazole :-IR $\vee \max (\mathrm{KBr}) \mathrm{cm}^{-1}: 3345(\mathrm{~N}-\mathrm{H}), 1685(\mathrm{C}=\mathrm{N}), 1602(\mathrm{C}=\mathrm{C}), 1234(\mathrm{C}-\mathrm{N}), 1078\left(-\mathrm{OCH}_{3}\right) .{ }^{1} \mathrm{H}$ NMR ( $\left.\mathrm{CDCl}_{3}, \delta \mathrm{ppm}\right): 5.08\left[\mathrm{~s}, 2 \mathrm{H}, \mathrm{OCH}_{2}\right], 2.37$ [s, 3H, $\left.\mathrm{CH}_{3}\right], 3.82\left[\mathrm{~s}, 3 \mathrm{H}, \mathrm{OCH}_{3}\right], 3.05$ [dd, J=17.0 Hz 
,1H, Ha], 3.74 [dd, J=16.91 Hz ,1H, Hb], 5.03 [dd, 1H, Hx] 6.86-7.85 [m, 16H, Ar-H]. MS ( m/z) : 483 $[\mathrm{M}]^{+}, \quad 468 \quad\left[\mathrm{C}_{29} \mathrm{H}_{25} \mathrm{ClN}_{2} \mathrm{O}_{2}\right]^{+}, 406 \quad\left[\mathrm{C}_{24} \mathrm{H}_{23} \mathrm{ClN}_{2} \mathrm{O}_{2}\right]^{+}, \quad 358 \quad\left[\mathrm{C}_{23} \mathrm{H}_{22} \mathrm{~N}_{2} \mathrm{O}_{2}\right]^{+}, \quad 270 \quad\left[\mathrm{C}_{18} \mathrm{H}_{22} \mathrm{O}\right]^{+}, \quad 262$ $\left[\mathrm{C}_{15} \mathrm{H}_{15} \mathrm{ClO}_{2}\right]^{+}, 238\left[\mathrm{C}_{17} \mathrm{H}_{18} \mathrm{O}\right]^{+}, 157\left[\mathrm{C}_{10} \mathrm{H}_{21} \mathrm{O}\right]^{+}, 142\left[\mathrm{C}_{7} \mathrm{H}_{7} \mathrm{ClO}\right]^{+}, 138\left[\mathrm{C}_{8} \mathrm{H}_{10} \mathrm{O}_{2}\right]^{+}, 120\left[\mathrm{C}_{9} \mathrm{H}_{12}\right]^{+}, 112$ $\left[\mathrm{C}_{6} \mathrm{H}_{5} \mathrm{Cl}\right]^{+}, 70\left[\mathrm{C}_{3} \mathrm{H}_{6} \mathrm{~N}_{2}\right]^{+}$.

(C-V) 3-(p-methoxyphenyl)-5-[4'-(p-chlorobenzyloxy)-3'-methoxyphenyl]-1-phenyl-4,5-dihydro-1Hpyrazole :-IR $v \max (\mathrm{KBr}) \mathrm{cm}^{-1}: 3355(\mathrm{~N}-\mathrm{H}), 1647(\mathrm{C}=\mathrm{N}), 1606(\mathrm{C}=\mathrm{C}), 1240(\mathrm{C}-\mathrm{N}), 1065\left(-\mathrm{OCH}_{3}\right) .{ }^{1} \mathrm{H}$ NMR $\left(\mathrm{CDCl}_{3}, \delta \mathrm{ppm}\right): 5.10\left[\mathrm{~s}, 2 \mathrm{H}, \mathrm{OCH}_{2}\right], 3.84\left[\mathrm{~s}, 3 \mathrm{H}, \mathrm{OCH}_{3}\right], 3.80\left[\mathrm{~s}, 3 \mathrm{H}, \mathrm{OCH}_{3}\right], 3.07$ [dd, J=17.01 Hz ,1H, Ha], 3.76 [dd, J=16.95 Hz ,1H, Hb], 5.07[dd, 1H, Hx] 6.83-7.95 [m, 16H, Ar-H]. MS ( m/z) : 498 $[\mathrm{M}]^{+}, \quad 468 \quad\left[\mathrm{C}_{29} \mathrm{H}_{25} \mathrm{ClN}_{2} \mathrm{O}_{2}\right]^{+}, 422 \quad\left[\mathrm{C}_{24} \mathrm{H}_{23} \mathrm{ClN}_{2} \mathrm{O}_{3}\right]^{+}, \quad 374 \quad\left[\mathrm{C}_{23} \mathrm{H}_{22} \mathrm{~N}_{2} \mathrm{O}_{3}\right]^{+}, \quad 270 \quad\left[\mathrm{C}_{18} \mathrm{H}_{22} \mathrm{O}\right]^{+}, \quad 262$ $\left[\mathrm{C}_{15} \mathrm{H}_{15} \mathrm{ClO}_{2}\right]^{+}, 254\left[\mathrm{C}_{17} \mathrm{H}_{18} \mathrm{O}_{2}\right]^{+}, 157\left[\mathrm{C}_{10} \mathrm{H}_{21} \mathrm{O}\right]^{+}, 142\left[\mathrm{C}_{7} \mathrm{H}_{7} \mathrm{ClO}\right]^{+}, 138\left[\mathrm{C}_{8} \mathrm{H}_{10} \mathrm{O}_{2}\right]^{+}, 136\left[\mathrm{C}_{9} \mathrm{H}_{12} \mathrm{O}\right]^{+}, 112$ $\left[\mathrm{C}_{6} \mathrm{H}_{5} \mathrm{Cl}\right]^{+}, 70\left[\mathrm{C}_{3} \mathrm{H}_{6} \mathrm{~N}_{2}\right]^{+}$.

\section{RESULT AND DISCUSSION}

Reported synthesis pyrazolines derivatives by the reaction between synthesized chalcones and phenylhydrazine using ethanol/alumina under microwave irradiation. In view of theses, we reported the condensation of synthesized substituted chalcone with phenylhydrazine using ethanol to yield novel pyrazoline derivatives containing benzyloxy pheny ring system under microwave irradiation . Microwave irradiation has been used to accelerate organic reactions because of high heating efficiency, providing remarkable rate enhancement, dramatic reduction in reaction times with improvement in yield and quality of products. Reactions that require hours or even days by conventional heating can often be accomplished in second or minutes by microwave heating. ${ }^{14}$ This technique has several advantages including clean reaction procedure, no need of catalyst, short reaction time and high yields of product. The obtained darivatives were characterized using spectroscopic technique, The IR spectra of pyrazoline darivatives show absence of carbonyl absorption band and the appearance of characteristic absorption band for $v$ $\mathrm{C}=\mathrm{N}$ at $1685-1647 \mathrm{~cm}^{-1}$ and a band at $1240-1233 \mathrm{~cm}^{-1}$ for $\mathrm{C}-\mathrm{N}$. Absorption bands at $3338-3355 \mathrm{~cm}^{-1}$ is characteristics of the $-\mathrm{NH}$ group.In the ${ }^{1} \mathrm{H}$ NMR spectrum, an $\alpha, \beta$, x pattern was observable, $\mathrm{H} \alpha, \mathrm{H} \beta$ and $\mathrm{Hx}$ appear as double doublets at $\delta 3.03-3.07,3.72-3.76$ and 5.01-5.10 ppm and aromatic protons appeared as multiplate at $\square 6.857 .95$ was observed.

\section{CONCLUSION}

In summary we reported the synthesis of novel pyrazoline derivatives containing benzyloxy phenyl ring system. Microwave induced Solution Phase/solid phase methods found to be excellent and convenient reaction route in terms of simple reaction procedure, quick reaction time giving percent yield of product as compared to conventional method.

\section{REFERENCES}

1- Hussein F. H. S.; Hawaiz F. E.; Azeez H. J. Hussein F. H. S.; Hawaiz F. E.; Azeez H. J. Synthesis and Characterization of Some New Pyrazoline Derivatives,4,373(2013).

2- Mogilaiah K., Reddy N.V.,Prashanthi M., Reddy G.R. and Reddy C.S., I. J. Hete.c Chem.,

11(4), 343(2002).

3- A.Dandia, R.Sngh and K.Arya I. J. Het. Chem., 11(4), 269(2002).

4- Mohammad S., Anees Ahamed S., Mohamed Ashraf A., Dharmarajan S., Perumal Y. Bioorg. Med.Chem.Lett. 16, 3947 (2006).

5- Rathish I.G., Javed K., Ahmad S., Bano S., Alam M. S., Pillai K. K.,Singh S., Bagchi V. 
Bioorg.Med.Chem.Lett. 19, 255 (2009).

6- Prasad Y.R., Rao A.L., Prasoona L., Murali K., Ravi Kumar P. Bioorg.Med.Chem.Lett. 15, 5030 (2005).

7- Banday A.H., Mir B.P., Lone I.H., Suri K.A. Steroids 75, 805 (2010).

8- Hudson B.J.F. and Lewis J.I., Food Chem., 10, 47 (1983).

9- Manna F., Chimenti F., Bolasco A., Rossi F. Cenicola M.L., Pharmacological research 26, 267 (1992).

10- Roelofav S.G., Arnold C. and Wellinga K., J. Agric. Food Chem., 27, 406 (1979).

11- Vornin V.G., Sharmova Z.I., Shachilova S. Ya., Kulikova L.D. and Zaks A.S., Khim. Farm. Zh., 19, 1208 (1985); Chem. Abstr., 104, 61668m (1986).

12- Hassan A.A., Regallia Egypt., J. Pharm. Sci., 29, 191 (1988).

13- Das N.B. and Mittra A.S., Indian J. Chem., 16B, 638 (1978).

14- Shinde A., Zangade S., Chavan S., Vibhute Y. Org.Commun. 7, 60 (2014). 